\title{
EPIDEMIOLOGY OF DERMATOMYCOSIS IN THE SUB-HIMALAYAN REGION OF WEST BENGAL, INDIA
}

\author{
Nibedita Haldar1, Niladri Haldar², Mani Kumar Sharma3 ${ }^{3}$ Goutam Dutta4, Nihar Ranjan Haldar ${ }^{5}$
}

\author{
${ }_{1}^{1}$ Professor, Department of Microbiology, MGM Medical College \& LSK Hospital, Kishanganj, Bihar. \\ ${ }^{2}$ RMO, "The Microbes Pathological Lab", Siliguri Dist., Darjeeling. \\ ${ }^{3}$ Consultant Dermatologist, Sharma Skin Foundation, Siliguri Dist., Darjeeling. \\ 4Senior Technician, "The Microbe Pathological Lab", Siliguri Dist., Darjeeling. \\ 5Professor, Department of General Medicine, Nobel Medical College \& Teaching Hospital Pvt Ltd., Biratnagar, Nepal.
}

\begin{abstract}
Dermatomycosis is the superficial infection of the keratinised tissues of the skin, hair and nail caused by various fungi including dermatophytes. This infection though trivial, has lot of psychological effect and requires effective treatment which is often costly. Numerous studies on clinico-mycological aspects of dermatomycosis have been conducted in different parts of India, but till now no reports are available on epidemiology of dermatomycosis from the Sub-Himalayan region of West Bengal. Over a period of two years (January 2013-December 2014) skin and nail samples were collected from 502 patients with signs and symptoms of dermatomycosis attending Sharma's skin foundation at Siliguri. Majority of the patients 301(60\%) were in the age group of 22-40 years. Males were more commonly affected than the females. The overall male to female ratio was 3: 1 . Tinea corporis was the commonest presentation no: 321(64\%). Direct microscopy of KOH mount, culture on Sabouraud dextrose agar (SDA) and identification of colony using Lactophenol cotton blue mount (LCB) has been done with all the specimens. Total no of skin and nail samples processed were $462(92 \%)$ and $40(8 \%)$ respectively. KOH mount was positive in $402(80 \%)$ of the total cases. In $80(16 \%)$ cases no fungal growth was found. Out of 422 culture positive cases Trichophyton (T) rubrum was the commonest fungi isolated $130(48.5 \%)$ followed by $\mathrm{T}$ mentagrophytes $54(20.1 \%)$. Other fungi isolated were Microsporum (M) gypseum 21(7.8\%) T verrucosum 23(8.6\%), T tonsurans 10(3.7\%), T violaceum 18(6.7\%), T schenlenii 4(1.5\%), Epidermophyton floccosum 6(2.2\%), Hortaea werneckii 1, M persicolor 1(0.4\%), T terrestre 1(0.4\%), Chrysosporium keratinophilum 1, Aspergillus 64, Candida 56, Penicillium 24, Mucor 7, Fusarium 1. Clinical response rate to oral Fluconazole was very less 53(20\%), whereas the response rate to oral Terbenafine was high 214 (80\%). Most of the patients responded very well to oral Itraconazole 254(95\%).
\end{abstract}

KEYWORDS: Sabouraud Dextrose Agar, Trichophyton, Microsporum, Lactophenol Cotton Blue.

HOW TO CITE THIS ARTICLE: Nibedita Haldar, Niladri Haldar, Mani Kumar Sharma, Goutam Dutta, Nihar Ranjan Haldar. "Epidemiology of Dermatomycosis in the Sub-Himalayan Region of West Bengal, India". Journal of Evolution of Medical and Dental Sciences 2015; Vol. 4, Issue 91, November 12; Page: 15619-15623, DOI: 10.14260/jemds/2015/2245.

INTRODUCTION: Dermatomycosis are the superficial infections of the keratinised tissues of the skin, hair and nail caused by various fungi, most important and commonest agents being dermatophytes. Dermatophytosis manifest itself in various clinical forms, notably Tinea corporis, Tinea cruris, Tinea capitis, Tinea incognito, onychomycosis to name a few.

The infecting fungi are distributed variedly in different geographical regions of the world. $(1,2)$ This distributions are not static and the range of species in some areas may change dramatically and quickly. Relevant clinicoepidemiological data of such cases are vary scanty from North Eastern part of India.(3) There is a lot of uniqueness in clinical presentation of dermatophytes and dramatic shift in range of infecting fungi, for example Tinea capitis in this area used to be mostly of non-inflammatory type earlier is now mostly seen to be of inflammatory type infected by zoophilic species.

The higher incidence of Tinea incognito in this region is associated with the use of topical potent steroids as cosmetic

Financial or Other, Competing Interest: None.

Submission 13-10-2015, Peer Review 14-10-2015,

Acceptance 30-10-2015, Published 10-11-2015.

Corresponding Author:

Dr. Nibedita Haldar,

Ramakrishna Road,

$1^{\text {st }}$ by Lane (II JK Dutta Sarani),

Ashrampara, Siliguri-734001, Darjeeling.

E-mail: nibeditaslg@yahoo.com

DOI:10.14260/jemds/2015/2245. for years together by the population at large. Tinea unguium is also very common and mostly found to be caused by $\mathrm{T}$ mentagrophyte and usually of DLSO (Distal and lateral sub ungual onychomycosis) clinical type. So the knowledge of the geographical distribution in clinico epidemiology goes a long way in planning preventive and treatment strategies in such cases on a regional basis.

Over the past decades, non-dermatophytes, as agents of superficial fungal infections in humans, produce lesions that are clinically similar to those caused by dermatophytic infections. These infections, though trivial, have lots of psychological effects and require effective treatment which is often costly. Therefore the present study was undertaken with a view to find out the prevalence of dermatophytes and non-dermatophytes and to identify the commonest pathogens of superficial fungal infections in Siliguri - a SubHimalayan region of West Bengal, India.

MATERIALS AND METHODS: A clinico-microbiological study was conducted from January 2013 upto December 2014 between Sharma's Skin Foundation and The Microbes Pathological lab at Siliguri, North Bengal. Skin and nail samples were collected from 502 patients of all age groups and both the sexes attending sharma's skin foundation at Siliguri with signs and symptoms of Dermatomycosis. These patients had not been treated earlier for the fungal infections.

Dermatomycosis was suspected when a lesion had central clearing with advancing, red, scally, elevated border, which might result in vesicles on the border of the affected area. A nail infection was suspected when there was distal 
hyperkeratosis, when chalky and dull yellow debris was found under the nail if it was separated from its bed, and the nail was brittle.

The skin scrapping were collected from the active edges of the lesion with a blunt scalpel after cleaning it thoroughly with $70 \%$ alcohol. The infected nails were clipped and processed as per standard mycological techniques.

A detailed history of the selected patients was taken regarding duration of illness, involvement of other sites, and history of similar episodes in the family members and immunocompromised condition.

Samples processing and further laboratory diagnosis done at "The Microbes" pathological lab, Siliguri. All the samples were subjected to direct microscopy in $10 \%$ potassium hydroxide (KOH) for skin samples and $40 \% \mathrm{KOH}$ for nail samples. The samples were cultured in SDA bottle into two sets, one group with chloramphenicol and other with cycloheximide (To prevent growth with saprophytic fungi and bacteria)along with choramphenicol and incubated at $37^{\circ} \mathrm{C}$ and $25^{\circ} \mathrm{C}$; respectively. The cultures were examined for presence of growth, colony morphology, and pigment production. LCB mount was done for the identification of the colony by microscopy. Slide cultures were also prepared for identification of specific fungal species.

RESULTS: A total number of 502(100\%) samples were screened during the study period of two years (January 2013 -Decemeber 2014) of which total number of skin samples and total number of nail samples processed were $462(92 \%)$ and $4(8 \%)$ respectively. Of the 502(100\%) samples, $402(80 \%)$ were confirmed by $\mathrm{KOH}$ microscopic examination and $422(84 \%)$ were culture positive. Total number of Dermatophytes isolated were 268(63.5\%) and those of non-dermatophytes isolated were $154(36.5 \%)$. Total number of patients in the age group of 1-22 years were $100(20 \%)$, in the age group of $22-40$ Years were $301(60 \%)$ and in the age group of 40-70 years were $101(20 \%)$. Overall male to female ratio were $3: 1$. Commonest clinical presentation were Tinea corporis: 321(64\%). Clinical response rate of the Dermatophytes to oral Fluconazole was $53(20 \%)$ whereas clinical response rate to oral Terbenafine was quite high: $214(80 \%)$. Most of the patients responded very well to oral Itraconazole: $254(95 \%)$

DISCUSSION: The prevalence of dermatophytosis is governed by environmental conditions, personal hygiene and individual's susceptibility from place to place. The isolation of different species of dermatophytes also varies markedly from one ecological niche to another depending on their primary natural habitat. Few studies have investigated the etiology of superficial fungal infections in the developing world, and consequently, there is less knowledge of changes in their epidemiology.

It is difficult to ascertain the reliability of the overall incidence and prevalence of various skin diseases caused by superficial mycoses in different parts of the world because incidence and prevalence figures may only be the representative of the population sampled, which may have associated risk factors for infection.(4)

In the present study skin was found to be the commonest site of Dermatomycosis followed by nail. Overall $\mathrm{T}$ rubrum was the chief isolate followed by $\mathrm{T}$ menta- grophytes from various parts of the skin \& nail as has been observed in other studies. $(5,6,7)$ However it is noteworthy that two uncommon species of the genus Trichophyton namely $\mathrm{T}$ schoenleinii and $\mathrm{T}$ terrestre were also identified in this study. In the presence study among the different species of the genus Microsporum $\mathrm{M}$ gypseum was found as the most common isolate $(7.8 \%)$ followed by $\mathrm{M}$ persicolor (0.4\%). Isolation of $\mathrm{M}$ gypseum could be accounted to patient's interaction with soil and domestic animals.(8)

Among the genus Epidermophyton Epidermophyton flocossum $(2.2 \%)$ was the only species isolated. The commonest age group of the patients were $22-40$ yrs. (60\%) which is in contrast to others studies.(2,5) The overall male to female ratio was $3: 1$. Higher incidence in males may be because they are exposed to outdoor with greater physical activity and more prone to trauma.(9) The percentage of nondermatophytic mould isolated was $36.5 \%$. The commonest non-dermatophyte isolated was Aspergillus in contrast to some other Indian studies showing Candida as the commonest non-dermatophytic mould.(7)

All the samples showing Aspergillus species in culture were also positive by direct $\mathrm{KOH}$ smear preparation. To rule out contamination telephonic conversation was also done with those patients whose samples showed growth of Aspergillus species and they were asked to come to the clinic for repeat sampling.

In about $75 \%$ of the cases repeat culture could be done which showed Aspergillus and in $25 \%$ of the cases patients did not come for repeat testing. A good no of patients $351(70 \%)$ gave history of household contact with cases $201(40 \%)$, contact with animals $100(20 \%)$ and history of trauma to nails $50(10 \%)$. This confirms that these infections can be tranmitted from person to person by sharing routine usable articles or fomites. $(10,11)$

People of lower socio-economic status with poor hygiene and overcrowding, some clinical conditions like Diabetes mellitus, Asthma etc. were the common prerequisite conditions. Most of the patients (62\%) came from rural areas with agriculture as their main occupation. 23\% people were urban dweller working in factories, shops or garages. Only $15 \%$ of the people belonged to higher socioeconomics status with jobs in offices, Schools and colleges. Cases were scattered throughout the year with highest incidence in rainy season (50\%) and summer (20\%).

Before starting the treatment for dermatomycosis, it is essential to establish the diagnosis of the disease so that specific therapeutic modalities can be adopted and monitored during the course of treatment. The clinician can now have trust that dermatophytic as well as nondermatophytic pathogens can be eradicated effectively if timely and proper diagnosis is made.

In the recent years, introduction of two new antifungal agents, itraconazole and terbinafine, have represented major advance in therapy for dermatomycosis. Both drugs are highly effective and have in large parts replaced use of griseofulvin in the treatment of onychomycosis mainly because of relatively low efficacy of griseofulvin. Itraconazole and terbinafine may be given as pulse therapy in patients of dermatophytosis for better results. The 'Clinical cure' is defined as disappearance of all lesions on each nail or residual disease of no more than $10 \%$ of original disease surface and 'mycological cure' is defined as negative culture and negative microscopy. 
Non-dermatophytic molds are difficult to eradicate. Itraconazole has recently been reported to be effective in nail infections caused by Aspergillus, Fusarium and Scopulariopsis brevicaulis with mycological and clinical cure rate upto about $88 \%$. The resistance to infection may be acquired after primary infection. It varies in duration and degree depending on host, site and species of fungus causing infection. In the present study clinical response rate of the dermatophytes to oral Fluconazole was only 20\%, but response rate to oral Terbenafine and oral Itraconazole was $80 \%$ and $95 \%$ respectively Though antifungal drugs should ideally be given on the basis of in vitro sensitivity of the isolate but it is cumbersome, still to be standardized for routine use and is possible only in very big institutes with all the modern facilities.

So identification of fungal species is vital to curtail the drug resistance among the fungi. Therefore direct microscopy along with culture and LCB mount of the fungal growth still remains as the methods of choice for the laboratory diagnosis of dermatomycosis in the periphery where molecular diagnosis are not available.(12)

\section{REFERENCES:}

1. Balakumar Srinivasan, Rajan Suyambu, Thirunalasundari Thiagarajan, Jeeva Solomon. Epidemiology of dermatophytosis in and around Tiruchirapally, Tamilnadu, India. Asian Pacific J Tropical Dis 2012; 2(4): 286- 289.

2. Madhavi S, Rama Rao MV, Jyothsna K. Mycological study of dermatophytosis in rural population. Annals of Biological Research 2011; 2(3): 88-93.

3. Sarma Smita, Borthakur AK. A clinico-epidemiological study of dermatophytosis in Northeast India. Indian J Dermatol Venereol Leprol 2007; 73(6): 427.
4. Chander Jagdish. Text book of Medical Mycology; 2009 (3rd edition): 123 -124.

5. Singla Bhavna, Malhotra Rubina, Walia Geeta. Mycological study of dermatophytosis in 100 clinical samples of skin hair and nail. International $\mathrm{J}$ of Pharmacy and Pharmaceutical sciences 2013; 5(4) 763- 765.

6. Prasad PVS, Priya K, Kaviarasan PK, Ananthi C, Sarayu LA. Study of chronic dermatophytic infection in a rural hospital. Ind j Dermatol Venereol Leprol 2005; 71 (2): 129- 130 .

7. Lakshmanan A, Ganeshkumar P, Raam Mohan S, Hemamalini M, Madhavan R. Epidemiological and clinical pattern of dermatomycoses in rural India. Indian J Medical Micro 2015; 33 (5): 134 - 136.

8. V M Ramesh, A Hilda. Mycopathologia 1998; 143: 139 145.

9. Jain Neetu, Sharma Meenakshi, Saxena VN. Clinicomycological study of dermatophytosis in Jaipur, Rajasthan. Indian J Dermatol Venereol Leprol 2008; 74(3): $274-275$.

10. SharmalNL, Gupta ML, Sharma RC, Singh P, Gupta N. Superficial mycoses in Shimla. Ind J Dermatol Venereol Leprol 1983; 49(6):266-269.

11. Patwardhan N, Dave R. Dermatophytosis in and around Aurangabad. Indian J Pathol Micro 1999; 42(4): 455 462.

12. Kannan P, Janaki C, Selvi G S. Prevalence of dermatophytes and other fungal agents isolated from clinical samples. Indian J Medical Micro 2006; 24(3): 212 -215 .

\begin{tabular}{|c|}
\hline Total KOH positive 402(80) \\
\hline KOH positive \& culture negative $40(8)$ \\
\hline KOH negative \& culture positive $30(6)$ \\
\hline Total culture positive $422(84)$ \\
\hline Both positive $400(79.7)$ \\
\hline Both negative $25(5)$ \\
\hline Table 1: Microscopy and culture positivity of 502 clinical samples: $n$ (\%) \\
\hline
\end{tabular}

\begin{tabular}{|c|c|c|c|c|c|c|c|c|c|c|c|}
\hline $\begin{array}{l}\text { Clinical } \\
\text { types }\end{array}$ & $\begin{array}{c}\text { T. } \\
\text { Rubrum }\end{array}$ & $\begin{array}{c}\text { T. } \\
\text { Mentagrophytes }\end{array}$ & $\begin{array}{c}\text { T. } \\
\text { verrucosum }\end{array}$ & $\begin{array}{c}\mathrm{T} . \\
\text { violaceum }\end{array}$ & $\begin{array}{c}\text { T. } \\
\text { tonsurans }\end{array}$ & $\begin{array}{c}\text { T. } \\
\text { schoenleinii }\end{array}$ & $\begin{array}{c}\text { T. } \\
\text { Terrestre }\end{array}$ & $\begin{array}{c}\text { M } \\
\text { gypserum }\end{array}$ & $\begin{array}{c}\mathbf{M} \\
\text { persicolor }\end{array}$ & $\begin{array}{l}\text { Epiderm- } \\
\text { ophyton } \\
\text { floccosum }\end{array}$ & Total \\
\hline T. unguium & 23 & 14 & 2 & 0 & 0 & 0 & 0 & 0 & 0 & 1 & $40(14.9)$ \\
\hline T. corporis & 52 & 14 & 19 & 14 & 10 & 2 & 1 & 20 & 1 & 5 & $138(51.5)$ \\
\hline T. Pedis & 19 & 10 & 1 & 0 & 0 & 1 & 0 & 0 & 0 & 0 & $31(11.6)$ \\
\hline T. cruris & 26 & 7 & 1 & 2 & 0 & 0 & 0 & 0 & 0 & 0 & $36(13.4)$ \\
\hline T. manum & 10 & 2 & 0 & 0 & 0 & 0 & 0 & 0 & 0 & 0 & $12(4.5)$ \\
\hline T. faciei & 0 & 7 & 0 & 2 & 0 & 1 & 0 & 1 & 0 & 0 & $11(4.1)$ \\
\hline Total & $130(48.5)$ & $54(20.1)$ & $23(8.6)$ & $18(6.7)$ & $10(3.7)$ & $4(1.5)$ & $1(0.4)$ & $21(7.8)$ & $1(0.4)$ & $6(2.2)$ & $268(100)$ \\
\hline
\end{tabular}




\begin{tabular}{|c|}
\hline Aspegillus species $64(41.6)$ \\
\hline Candida species $56(36.4)$ \\
\hline Penicillium species $24(15.6)$ \\
\hline Mucor $7(4.6)$ \\
\hline Fusarium $1(0.6)$ \\
\hline Chrysosporium keratinophilum $1(0.6)$ \\
\hline Hortaea werneckii $1(0.6)$ \\
\hline Total 154(100) \\
\hline Table 3: Distribution of non-dermatophytic moulds $\mathbf{n}=(\%)$ \\
\hline
\end{tabular}
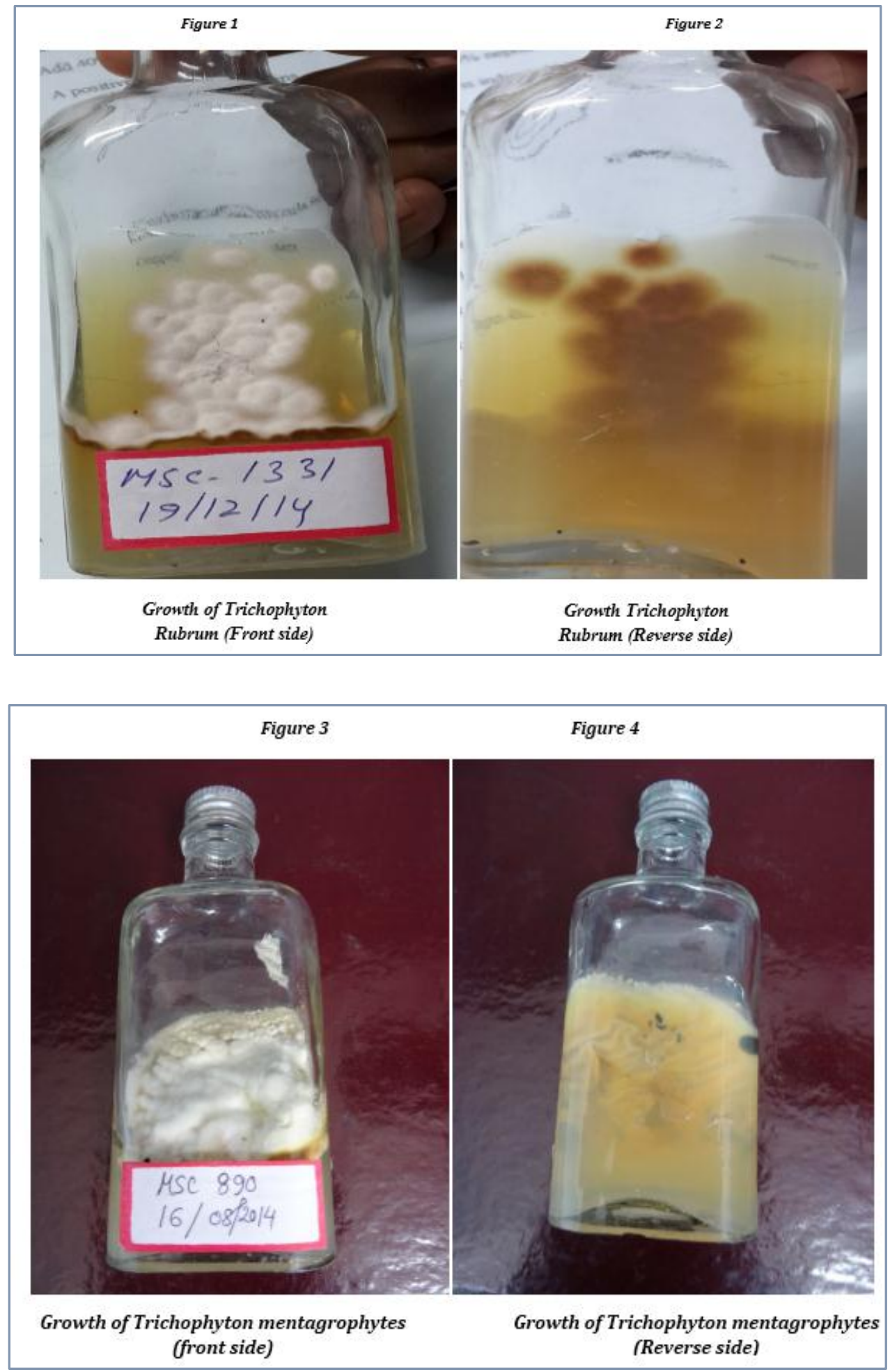

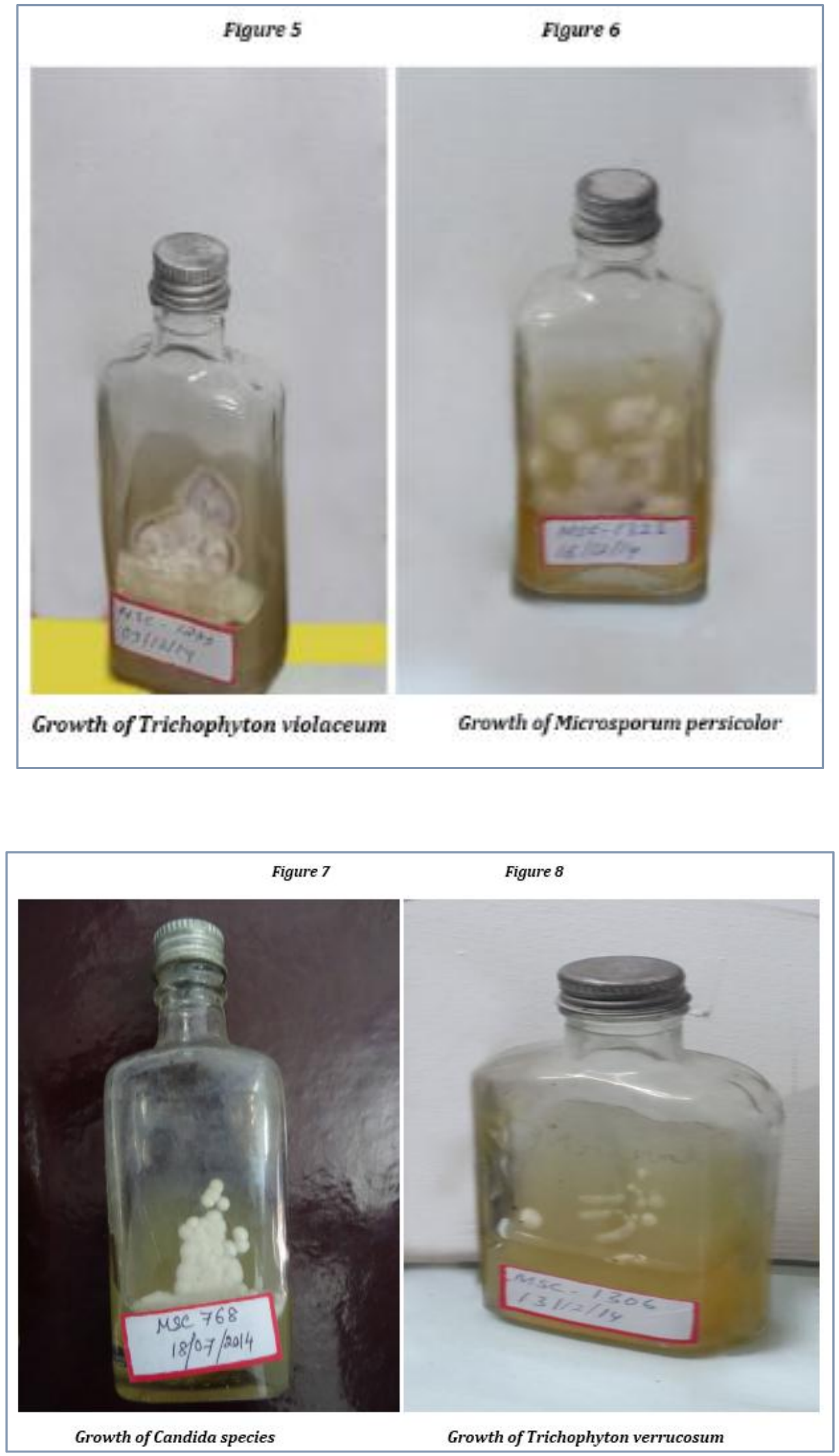